\title{
A notificação de eventos adversos e suas lacunas no processo da segurança do paciente
}

RESUMO | Objetivo: analisar as contribuições da notificação de eventos adversos para a segurança do paciente e os entraves no processo de cuidar que prejudicam a oferta de uma assistência hospitalar segura. Métodos: trata-se de uma revisão integrativa da literatura nas bases de dados SciELO, LILACS e BDENF. Para a definição da pergunta norteadora utilizou-se a estratégia PICO e quanto ao processo de seleção dos estudos, utilizou-se o fluxograma PRISMA. As buscas abrangeram o período de 2014 a 2019, sendo selecionados os artigos disponíveis em português, na íntegra e no formato original. Resultados: Foram selecionados 16 artigos para análise, sendo evidenciado que a identificação das lacunas no processo de trabalho pode fornecer elementos importantes para promoção da segurança na assistência à saúde. Conclusão: a notificação dos eventos adversos proporciona um cuidado mais seguro, pois possibilitam a análise dos incidentes, identificação das causas e a implantação de estratégias para minimização dos erros evitáveis.

Palavras-chaves: Segurança do Paciente; Enfermagem; Qualidade da Assistência à Saúde; Notificação.

\begin{abstract}
I Objective: to analyze the contributions of the notification of adverse events to patient safety and the obstacles in the care process that hinder the offer of safe hospital care. Methods: it is an integrative literature review in the databases SciELO, LILACS and BDENF. For the definition of the guiding question, the PICO strategy was used and for the study selection process, the PRISMA flowchart was used. The searches covered the period from 2014 to 2019, with articles available in Portuguese, in full and in the original format being selected. Results: 16 articles were selected for analysis, showing that the identification of gaps in the work process can provide important elements for promoting safety in health care. Conclusion: the notification of adverse events provides safer care, as they allow the analysis of incidents, identification of causes and the implementation of strategies to minimize avoidable errors.
\end{abstract}

Keywords: Patienty Safety; Nursing; Quality of Health Care; Notifications.

RESUMEN | Objetivo: analizar las contribuciones de la notificación de eventos adversos a la seguridad del paciente y los obstáculos en el proceso de atención que obstaculizan la oferta de atención hospitalaria segura. Métodos: es una revisión bibliográfica integradora en las bases de datos SciELO, LILACS y BDENF. Para la definición de la pregunta guía, se utilizó la estrategia PICO y para el proceso de selección de los estudios, se utilizó el diagrama de flujo PRISMA. Las búsquedas abarcaron el período de 2014 a 2019, con artículos disponibles en portugués, en su totalidad y en el formato original seleccionado. Resultados: se seleccionaron 16 artículos para su análisis, que muestran que la identificación de brechas en el proceso de trabajo puede proporcionar elementos importantes para promover la seguridad en la atención de la salud. Conclusión: la notificación de eventos adversos brinda una atención más segura, ya que permiten el análisis de incidentes, la identificación de causas y la implementación de estrategias para minimizar los errores evitables.

Palabras claves: Seguridad del Paciente; Enfermería; Calidad de la Atención de Salud; Notificación.

\section{Carolina Catarine Maciano de S. Moraes}

Enfermeira. Universidade Paulista (UNIP), Campus Brasília-DF, Brasil.

ORCID: 0000-0002-4456-839X

\section{Ricardo Saraiva Aguiar}

Professor Assistente. Curso de Graduação em Enfermagem, Universidade Paulista (UNIP), Campus Brasília, Distrito Federal, Brasil.

ORCID: 0000-0003-0335-2194

Recebido em: 19/07/2020

Aprovado em: 28/07/2020

INTRODUÇÃO

$\Lambda$ segurança do paciente é um dos fundamentos essenciais na assistência à saúde e um elemento indispensável para gestão da qualidade em âmbito mundial ${ }^{1}$. Essa tem sido um assunto de debate entre gestores e equipes de saúde a nível global e está mais presente nos roteiros das reuniões de instituições de saúde públicas e privadas depois da publicação do relatório americano "Errar é humano: construindo um sistema de saúde mais seguro" do Instituto de Medicina ${ }^{2-3}$. O documento aponta a ocorrência de iatrogenias em um pouco mais de $3 \%$ do total de prontuários analisados. Além disso, os autores referiram o óbito de 44.000 a 98.000 pacientes por ano nos Estados Unidos da América (EUA), cujo as causas foram incidentes, que poderiam, a maior parte, ser evitados ${ }^{2}$.

Diante disso, para prevenir danos aos pacientes, é necessário captar falhas antes que ocorram, através da associação de métodos que detalhem os processos complexos ${ }^{4}$. Assim, políticas e procedimentos são implantados para proteger os pacientes de danos. Entretanto, um entendimento mais profundo do "porquê" um evento adverso (EA) específico ocorreu, focando menos no indivíduo que cometeu o erro, pode ter resultados positivos para a cultura de segurança. Parte-se, assim, do pressuposto que existem lacunas no que concerne à execução efetiva das atividades gerenciais e assistenciais ${ }^{5}$. 
Nessa perspectiva, é necessário promover o fortalecimento da cultura de segurança, visto que ela proporciona a percepção do erro como algo que ocorre por diversas causas e a cultura positiva possibilita aos serviços de saúde identificar e gerir circunstâncias e/ou cenários com potenciais fatores de risco, conduzindo ações de melhorias no cuidado prestado e evitando que a falha aconteça outra vez a fim de garantir a segurança do paciente ${ }^{6-9}$.

Não obstante, revela-se a notificação espontânea como uma relevante ferramenta para a detecção de incidentes em saúde, por ser uma metodologia de baixo custo, abranger profissionais que prestam cuidados ao paciente, e alertar para a promoção da segurança no âmbito hospitalar, além de ser um indicador para o gerenciamento da qualidade dos serviços ${ }^{10}$.

Sob essa perspectiva, destaca-se a questão que norteou a presente proposta investigativa: como a notificação de eventos adversos pode contribuir para segurança do paciente e quais são os entraves para oferta de uma assistência hospitalar segura? Diante disso, esse estudo busca analisar as contribuições da notificação de eventos adversos para a segurança do paciente e os entraves no processo de cuidar que prejudicam a oferta de uma assistência hospitalar segura.

\section{MÉTODOS}

Trata-se de uma revisão integrativa da literatura, uma vez que contribui no processamento sistemático e analítico dos resultados, pois é característica da busca de informações sobre um assunto ou tema que resuma a situação da ciência sobre um problema de pesquisa, visando a clareza do determinado tema ${ }^{11}$.

Elaborou-se, na primeira fase, a pergunta norteadora de pesquisa. Para essa construção, utilizou-se da estratégia PICO: $\mathrm{P}$ - população e problema; I - intervenção; C - comparação; e O - outcome (termo em inglês que signi- fica desfecho $)^{12}$. Assim, considerou-se P: pacientes hospitalizados; I: notificação de eventos adversos e os entraves no processo de trabalho; C: qualquer comparação relacionada a notificação de eventos adversos e aos entraves no processo de trabalho; O: segurança do paciente. Nesta direção, a pergunta construída foi: como a notificação de eventos adversos pode contribuir para segurança do paciente e quais são os entraves para oferta de uma assistência hospitalar segura?

Construiu-se para a segunda fase, uma estratégia de busca por dois revisores independentes utilizando os descritores segurança do paciente, enfermagem e qualidade da assistência à saúde nas bases de dados eletrônicas Scientific Electronic Library Online (SCIELO), Literatura Científica e Técnica da América Latina e Caribe (LILACS) e Base de Dados em Enfermagem (BDENF).

Refinou-se, contemplando a terceira fase da pesquisa com a aplicação dos critérios de inclusão previamente estabelecidos na estratégia de busca: artigos publicados de forma online nos últimos 6 anos (2014 a 2019); disponíveis em língua portuguesa; na íntegra; e no formato original oriundos de produções científicas diversificadas.

Leram-se criticamente, na quarta fase, os resumos dos estudos recuperados, excluindo os duplicados e aqueles cujo objetivo, resultados ou conclusão não mencionavam sobre a notificação de eventos adversos e os entraves no processo de trabalho em ambiente hospitalar.

A extração dos dados foi conduzida por dois revisores independentes, sendo que os desacordos entre os revisores em relação aos dados extraídos eram discutidos, tendo como referência a publicação original.

\section{RESULTADOS}

Apresenta-se, na figura 1, o fluxograma descritor dos resultados obtidos a partir da estratégia de busca de acordo com o fluxograma PRISMA13.

Por conseguinte, no quadro 1 estão apresentadas as informações a respeito dos 16 artigos contidos nesta revisão integrativa. Foram interpretados e sintetizados todos os resultados, através de uma comparação dos dados evidenciados na análise dos artigos.

Resultou-se a busca na literatura um
Figura 1 - Fluxograma da seleção dos estudos. Brasília, Distrito Federal, Brasil, 2019.

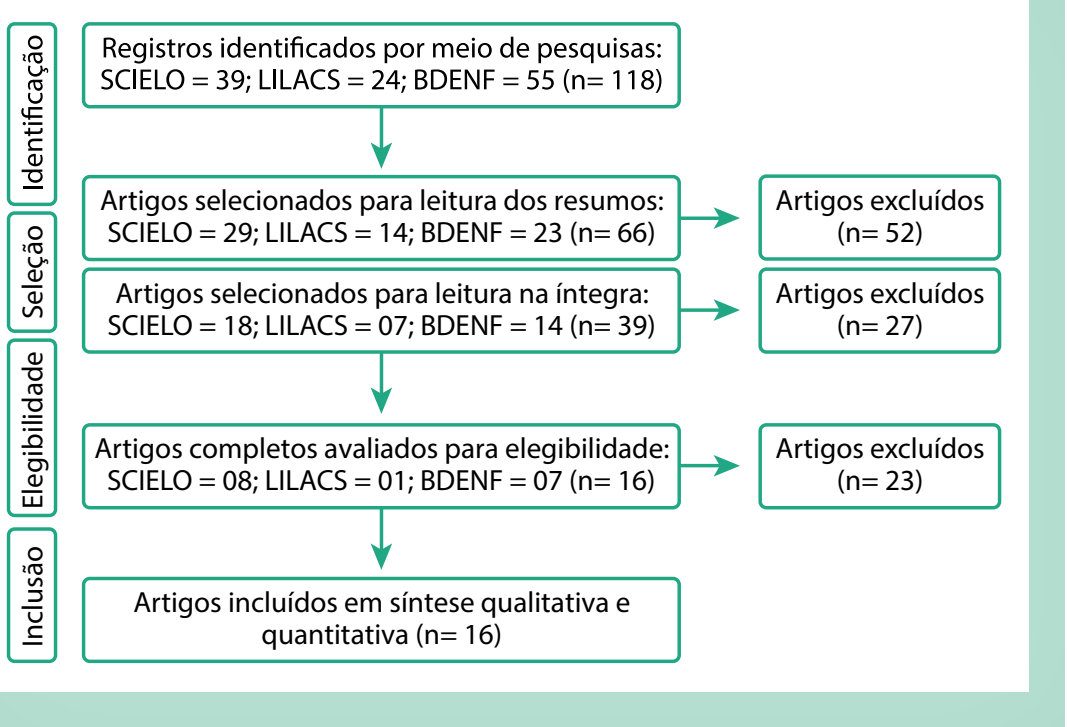


total de 118 artigos capturados e, desses, 39 estavam no SCIELO, 24 na LILACS e 55 artigos na BDENF. Reduziu-se, a partir da aplicação dos filtros de inclusão, o número de ocorrência: no SCIELO, recu- peraram-se 29 (44\%) estudos; na LILACS, 14 (21,2\%); e na BDENF, 23 (34,8\%) estudos. Totalizaram-se 66 artigos submetidos à leitura dos resumos e à aplicação dos critérios de exclusão, gerando-se a rejeição de 27 artigos. Após a leitura completa dos artigos foram rejeitados ainda 23 artigos por não responderam à questão de pesquisa. Constituiu-se assim a amostra revisada de 16 artigos.

\begin{tabular}{|c|c|c|c|c|c|c|}
\hline & $\begin{array}{l}\text { Periódico, país } \\
\text { e ano de publi- } \\
\text { cação }\end{array}$ & Autor(es) & Título & Delineamento & Resultados & $\begin{array}{l}\text { Nível de } \\
\text { Evidência }\end{array}$ \\
\hline 은 & $\begin{array}{c}\text { Revista Brasileira } \\
\text { de Enfermagem, } \\
\text { Brasil, } 2019\end{array}$ & $\begin{array}{l}\text { Lima Gomes AT, } \\
\text { Ferreira Júnior } \\
\text { MA, Salvador } \\
\text { PTCO, Bezerril } \\
\text { MS, Chiavone } \\
\text { FBT, Santos VEP }\end{array}$ & $\begin{array}{l}\text { Segurança do pa- } \\
\text { ciente em situação } \\
\text { de emergência: per- } \\
\text { cepções da equipe } \\
\text { de enfermagem }\end{array}$ & $\begin{array}{l}\text { Estudo descritivo e misto, } \\
\text { realizado por meio de grupo } \\
\text { focal e técnicas projetivas. A } \\
\text { amostra foi composta por sete } \\
\text { profissionais de enfermagem. } \\
\text { A análise dos dados ocorreu } \\
\text { por meio dos softwares } \\
\text { Interface de R pour Analyses } \\
\text { Multidimensionnelles de } \\
\text { Textes et de Questionneires e } \\
\text { SPSS 22.0. }\end{array}$ & $\begin{array}{l}\text { Os aspectos imprescindíveis } \\
\text { para a garantia de um cuidado } \\
\text { seguro ao paciente são: } \\
\text { adequação do ambiente e } \\
\text { organizaçào do setor no que diz } \\
\text { respeito à exclusividade de lei- } \\
\text { tos para pacientes em situação } \\
\text { de emergências traumáticas; } \\
\text { disponibilidade de recursos } \\
\text { humanos e materiais em qua- } \\
\text { lidade e quantidade suficiente; } \\
\text { segurança durante o transporte } \\
\text { do paciente, com vistas aos } \\
\text { cuidados adequados para a } \\
\text { prevenção de quedas; o uso de } \\
\text { rotinas e protocolos no setor; e } \\
\text { a identificação e organização do } \\
\text { leito previamente à chegada do } \\
\text { paciente ao hospital. }\end{array}$ & IV \\
\hline 梁 & $\begin{array}{l}\text { Revista de Enfer- } \\
\text { magem da UFPE, } \\
\text { Brasil, } 2019\end{array}$ & $\begin{array}{c}\text { Pereira MCC, } \\
\text { Castro SFF, Brito } \\
\text { ES, Carvalho NV, } \\
\text { Pinheiro JDS, } \\
\text { Schneider KN- } \\
\text { LAG, Lavôr TSL }\end{array}$ & $\begin{array}{l}\text { Saberes e práticas } \\
\text { do enfermeiro na } \\
\text { unidade de terapia } \\
\text { intensiva }\end{array}$ & $\begin{array}{l}\text { Trata-se de estudo qualitativo, } \\
\text { descritivo, observacional, } \\
\text { realizado com dez enfermeiros } \\
\text { efetivos da UTI. Aplicou-se um } \\
\text { roteiro de entrevista semies- } \\
\text { truturado e analisaram-se os } \\
\text { dados por meio de Análise de } \\
\text { Conteúdo. }\end{array}$ & $\begin{array}{l}\text { Percebeu-se que o trabalho } \\
\text { aponta diferentes visões refe- } \\
\text { rentes ao conhecimento do en- } \\
\text { fermeiro sobre a qualidade da } \\
\text { assistência de Enfermagem na } \\
\text { UTI, porém, é possível definir } \\
\text { que eles atribuem a qualidade } \\
\text { da assistência ao cuidado } \\
\text { centrado no paciente, sendo } \\
\text { referidas outras vertentes como } \\
\text { a humanização, o holismo e a } \\
\text { segurança do paciente. }\end{array}$ & IV \\
\hline $\begin{array}{l}m \\
\text { 은 } \\
\frac{1}{4}\end{array}$ & $\begin{array}{c}\text { Revista CuidArte } \\
\text { Enfermagem, } \\
\text { Brasil, } 2019\end{array}$ & $\begin{array}{c}\text { Moraes AIS, } \\
\text { Santos VL, Paes } \\
\text { LBO, Parro MC }\end{array}$ & $\begin{array}{c}\text { Qualidade e } \\
\text { segurança na área } \\
\text { da saúde materno } \\
\text { infantil: avaliação de } \\
\text { eventos adversos }\end{array}$ & $\begin{array}{l}\text { Estudo transversal, descritivo } \\
\text { e quantitativo, obtido por } \\
\text { coleta de dados dos eventos } \\
\text { adversos notificados. O levan- } \\
\text { tamento de notificações do } \\
\text { setor maternidade no Núcleo } \\
\text { de Segurança do Paciente } \\
\text { ocorreu no período de maio } \\
\text { de } 2017 \text { a maio de } 2018 \text {. }\end{array}$ & $\begin{array}{l}\text { Ocorreram } 89 \text { notificações de } \\
\text { eventos adversos, sendo o even- } \\
\text { to trauma mamilar evidenciado } \\
\text { em } 30,3 \% \text { das notificações, se- } \\
\text { guido de falha na comunicação; } \\
\text { administração de medicamen- } \\
\text { tos e identificação, com } 25,8 \% \text {; } \\
20,2 \% \text { e } 14,6 \% \text {, respectivamen- } \\
\text { te. Os eventos adversos queda } \\
\text { e hemorragia surgiram com o } \\
\text { mesmo percentual de } 3,3 \% \text { e a } \\
\text { flebite em } 2,2 \% \text {. }\end{array}$ & IV \\
\hline
\end{tabular}




\begin{tabular}{|c|c|c|c|c|c|c|}
\hline 荥 & $\begin{array}{c}\text { Revista de } \\
\text { Pesquisa: Cuidado } \\
\text { é Fundamental } \\
\text { Online, Brasil, } \\
2019\end{array}$ & $\begin{array}{c}\text { Picolotto A, Ba- } \\
\text { rella D, Moraes } \\
\text { FR, Gasperi P }\end{array}$ & $\begin{array}{l}\text { A cultura de segu- } \\
\text { rança do paciente } \\
\text { da equipe de } \\
\text { enfermagem de um } \\
\text { ambulatório central }\end{array}$ & $\begin{array}{c}\text { Estudo de abordagem } \\
\text { quantitativa do tipo Survey } \\
\text { transversal. A coleta dos da- } \\
\text { dos foi realizada utilizando o } \\
\text { questionário Safety Attitudes } \\
\text { Questionnaire. }\end{array}$ & $\begin{array}{l}\text { Nenhuma das dimensões alcan- } \\
\text { çou a média mínima ( } 75 \text { pontos) } \\
\text { para uma cultura de segurança } \\
\text { do paciente adequada. }\end{array}$ & IV \\
\hline 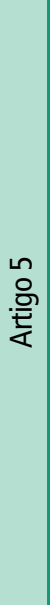 & $\begin{array}{l}\text { Revista Brasileira } \\
\text { de Enfermagem, } \\
\text { Brasil, } 2018\end{array}$ & $\begin{array}{l}\text { Gutierres LS, } \\
\text { Santos JLG, Pei- } \\
\text { ter CC, Menegon } \\
\text { FHA, Sebold LF, } \\
\text { Erdmann AL }\end{array}$ & $\begin{array}{l}\text { Boas práticas } \\
\text { para segurança } \\
\text { do paciente em } \\
\text { centro cirúrgico: } \\
\text { recomendações de } \\
\text { enfermeiros }\end{array}$ & $\begin{array}{l}\text { Pesquisa quanti-qualitativa, } \\
\text { do tipo descritiva e explora- } \\
\text { tória, desenvolvida a partir } \\
\text { de um Survey on line com } \\
220 \text { enfermeiros de centro } \\
\text { cirúrgico de diferentes regiões } \\
\text { do Brasil. O processamento } \\
\text { dos dados para analise textual } \\
\text { foi realizado pelo software } \\
\text { IRAMUTEQ. }\end{array}$ & $\begin{array}{l}\text { Obtiveram-se oito recomenda- } \\
\text { ções: (1) Envolvimento da equipe } \\
\text { multiprofissional e dos gestores } \\
\text { da instituição; (2) Estabelecimen- } \\
\text { to de uma cultura de segurança } \\
\text { do paciente; (3) Utilização do } \\
\text { checklist de cirurgia segura; } \\
\text { (4) Melhoria da comunicação } \\
\text { interpessoal; (5) Ampliação da } \\
\text { atuação do enfermeiro; (6) Dispo- } \\
\text { nibilidade adequada de recursos } \\
\text { físicos, materiais e humanos; (7) } \\
\text { Busca individual por atualização } \\
\text { profissional; e (8) Desenvolvi- } \\
\text { mento de ações de educação } \\
\text { continuada. }\end{array}$ & IV \\
\hline $\begin{array}{l}0 \\
\text { O } \\
.0 \\
\frac{0}{4}\end{array}$ & $\begin{array}{l}\text { Revista Brasileira } \\
\text { de Enfermagem, } \\
\text { Brasil, } 2018\end{array}$ & $\begin{array}{l}\text { Figueiredo ML, } \\
\text { Silva CSO, Brito } \\
\text { MFSF, D'Innocen- } \\
\text { zo M }\end{array}$ & $\begin{array}{l}\text { Análise da ocorrên- } \\
\text { cia de incidentes } \\
\text { notificados em } \\
\text { hospital-geral }\end{array}$ & $\begin{array}{c}\text { Estudo retrospectivo, descri- } \\
\text { tivo, quantitativo, realizado } \\
\text { em hospital-geral de Montes } \\
\text { Claros-MG. A amostra foi } \\
\text { composta de } 1.316 \text { incidentes } \\
\text { notificados entre } 2011 \text { a } 2014 . \\
\text { Os dados foram submetidos } \\
\text { à estatística descritiva no } \\
\text { software Statistical Package } \\
\text { for the Social Sciences versão } \\
18.0 .\end{array}$ & $\begin{array}{l}\text { A prevalência de incidentes } \\
\text { foi de } 33,8 \text { por } 1.000 \text { interna- } \\
\text { ções, tendo sido evidenciados } \\
\text { aumento ao longo do período } \\
\text { investigado e maior frequência } \\
\text { nas unidades de internação, no } \\
\text { setor de emergência e centro } \\
\text { cirúrgico. Houve maior ocorrência } \\
\text { em clientes adultos e relativos à } \\
\text { cadeia medicamentosa. As prin- } \\
\text { cipais causas foram o descumpri- } \\
\text { mento da rotina/protocolo, sendo } \\
\text { necessárias mudanças na rotina } \\
\text { e no treinamento. }\end{array}$ & IV \\
\hline 高 & $\begin{array}{l}\text { Revista de Enfer- } \\
\text { magem da UFPE, } \\
\text { Brasil, } 2018\end{array}$ & $\begin{array}{l}\text { Silva AT, Camelo } \\
\text { SHH, Terra FS, } \\
\text { Dázio EMR, } \\
\text { Sanches RS, } \\
\text { Resck ZMR }\end{array}$ & $\begin{array}{c}\text { Segurança do } \\
\text { paciente e a atuação } \\
\text { do enfermeiro em } \\
\text { hospital }\end{array}$ & $\begin{array}{l}\text { Estudo qualitativo, descritivo, } \\
\text { realizado com } 42 \text { enfermeiros } \\
\text { de uma instituição hospitalar. } \\
\text { Os dados foram reunidos a } \\
\text { partir de entrevista semiestru- } \\
\text { turada e submetidos à técnica } \\
\text { da hermenêutica dialética. }\end{array}$ & $\begin{array}{l}\text { Verificou-se que a utilização de } \\
\text { estratégias, como a comunicação, } \\
\text { a educação permanente e a } \\
\text { participação do acompanhante } \\
\text { foram essenciais para o fortaleci- } \\
\text { mento da segurança do paciente } \\
\text { na instituição. Já a superlotação } \\
\text { e a sobrecarga de trabalho foram } \\
\text { apresentadas como prejudiciais à } \\
\text { sua atuação. }\end{array}$ & IV \\
\hline 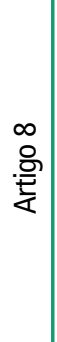 & $\begin{array}{l}\text { Revista de Enfer- } \\
\text { magem da UFPE, } \\
\text { Brasil, } 2018\end{array}$ & $\begin{array}{l}\text { Mazzoni VG, } \\
\text { Bittencourt LP, } \\
\text { Ribeiro ML, } \\
\text { Gouvêa MV }\end{array}$ & $\begin{array}{l}\text { Desafios da dimen- } \\
\text { são organizacional } \\
\text { do cuidado no coti- } \\
\text { diano de trabalhado- } \\
\text { res de enfermagem }\end{array}$ & $\begin{array}{l}\text { Estudo qualitativo, descritivo, } \\
\text { com } 18 \text { profissionais de um } \\
\text { Hospital Público Oncológico. } \\
\text { Os dados foram reunidos a } \\
\text { partir de observação partici- } \\
\text { pante e entrevista, com base } \\
\text { em roteiro semiestruturado, } \\
\text { e submetidos à Análise de } \\
\text { Conteúdo. }\end{array}$ & $\begin{array}{c}\text { Houve diferenças de percepção, } \\
\text { entre trabalhadores da gestão e } \\
\text { da assistência, com relação aos } \\
\text { desafios da dimensão organiza- } \\
\text { cional do cuidado; os principais } \\
\text { desafios estão relacionados à } \\
\text { resolutividade das ações propos- } \\
\text { tas referentes à segurança dos } \\
\text { pacientes. }\end{array}$ & IV \\
\hline
\end{tabular}




\begin{tabular}{|c|c|c|c|c|c|c|}
\hline 哭 & $\begin{array}{l}\text { Acta Paulista de } \\
\text { Enfermagem, } \\
\text { Brasil, } 2017\end{array}$ & $\begin{array}{c}\text { Ortega DB, D'In- } \\
\text { nocenzo M, Silva } \\
\text { LM, Bohomol E }\end{array}$ & $\begin{array}{l}\text { Análise de eventos } \\
\text { adversos em pacien- } \\
\text { tes internados em } \\
\text { unidade de terapia } \\
\text { intensiva }\end{array}$ & $\begin{array}{c}\text { Foi realizado um estudo } \\
\text { transversal, prospectivo, com } \\
\text { abordagem quantitativa, em } \\
304 \text { pacientes consecutivos } \\
\text { internados em Unidade de } \\
\text { Terapia Intensiva geral de um } \\
\text { hospital privado, admitidos } \\
\text { entre setembro e dezembro } \\
\text { de } 2013 \text {. }\end{array}$ & $\begin{array}{l}\text { Ocorreram } 39 \text { eventos adversos } \\
\text { sendo a lesão por pressão a mais } \\
\text { prevalente. Os pacientes que } \\
\text { apresentaram algum evento tiveram } \\
\text { maior média de idade, maior } \\
\text { prevalência de internações clínicas, } \\
\text { internações mais prolongadas, } \\
\text { maior escala Acute Physiology and } \\
\text { Chronic Health Evaluation (APACHE) } \\
\text { II, maior pontuação do Nursing } \\
\text { Activities Score (NAS), menor } \\
\text { escore na escala de Braden e menor } \\
\text { escala de Glasgow e não tiveram } \\
\text { diferenças significantes em relação } \\
\text { ao dimensionamento da equipe de } \\
\text { enfermagem. }\end{array}$ & IV \\
\hline 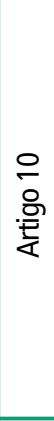 & $\begin{array}{c}\text { Revista da Escola } \\
\text { de Enfermagem } \\
\text { da USP, Brasil, } \\
2017\end{array}$ & $\begin{array}{c}\text { Siman AG, } \\
\text { Cunha SGS, Brito } \\
\text { MJM }\end{array}$ & $\begin{array}{l}\text { A prática de noti- } \\
\text { ficação de eventos } \\
\text { adversos em um } \\
\text { hospital de ensino }\end{array}$ & $\begin{array}{l}\text { Estudo de caso qualitativo, } \\
\text { realizado em um hospital } \\
\text { de ensino com participantes } \\
\text { do Núcleo de Segurança } \\
\text { do Paciente e a equipe de } \\
\text { Enfermagem. A coleta de } \\
\text { dados foi realizada por meio } \\
\text { de entrevistas, observação e } \\
\text { pesquisa documental para } \\
\text { tratamento dos dados sob a } \\
\text { Análise de Conteúdo. }\end{array}$ & $\begin{array}{l}\text { A prática de notificação de eventos } \\
\text { adversos encontrava-se marcada } \\
\text { pelo medo e apresentava lacunas } \\
\text { no conhecimento. Falta melhor } \\
\text { relação entre líderes, membros do } \\
\text { Núcleo de Segurança do Paciente e } \\
\text { demais profissionais para que todos } \\
\text { assumam efetivamente seus papeis } \\
\text { sociais no âmbito da segurança do } \\
\text { paciente no hospital. }\end{array}$ & IV \\
\hline 吾 & $\begin{array}{l}\text { Cogitare Enferma- } \\
\text { gem, Brasil, } 2017\end{array}$ & $\begin{array}{c}\text { Ferezin TPM, Ra- } \\
\text { mos D, Caldana } \\
\text { G, Gabriel CS, } \\
\text { Bernardes A }\end{array}$ & $\begin{array}{l}\text { Análise da notifica- } \\
\text { ção de eventos ad- } \\
\text { versos em hospitais } \\
\text { Acreditados }\end{array}$ & $\begin{array}{l}\text { Estudo descritivo, tipo Survey, } \\
\text { transversal, com abordagem } \\
\text { quantitativa, realizado em } \\
\text { três hospitais acreditados } \\
\text { do interior do estado de São } \\
\text { Paulo, com amostra de } 61 \\
\text { enfermeiros e } 250 \text { técnicos e } \\
\text { auxiliares de enfermagem. A } \\
\text { coleta de dados ocorreu entre } \\
\text { novembro de } 2014 \text { e abril de } \\
2015 .\end{array}$ & $\begin{array}{l}\text { Demonstrou-se que } 126(75,4 \%) \\
\text { participantes possuíam conheci- } \\
\text { mento para realizar notificação de } \\
\text { eventos adversos, mas apenas } 109 \\
\text { (65,3\%) relataram ter autorização } \\
\text { para realizá-las, sendo os enfermei- } \\
\text { ros apontados pelos participantes } \\
\text { como responsáveis por esta ação. } \\
\text { Do total de participantes, } 76 \\
\text { (45,5\%) afirmaram que a notifi- } \\
\text { cação dos eventos gera medidas } \\
\text { punitivas para os profissionais } \\
\text { envolvidos. Apesar disso, } 62 \text { (37,1\%) } \\
\text { não destacaram medo de punição } \\
\text { como fator dificultador. }\end{array}$ & IV \\
\hline$\frac{2}{\frac{8}{2}}$ & $\begin{array}{c}\text { Revista de Enfer- } \\
\text { magem da UFPE, } \\
\text { Brasil, } 2017\end{array}$ & $\begin{array}{l}\text { Pena MM, } \\
\text { Melleiro MM }\end{array}$ & $\begin{array}{l}\text { O método de análise } \\
\text { de causa raiz para } \\
\text { a investigação de } \\
\text { eventos adversos }\end{array}$ & $\begin{array}{l}\text { Estudo quantitativo, explora- } \\
\text { tório e descritivo, com coleta } \\
\text { retrospectiva dos dados, em } \\
\text { hospital universitário, utilizan- } \\
\text { do-se uma amostra de } 263 \\
\text { eventos. A análise empregou } \\
\text { estatística descritiva e testes } \\
\text { específicos. }\end{array}$ & $\begin{array}{l}39,9 \% \text { correspondem a flebites; } \\
32,7 \% \text { a erros de medicaçãa; } 16 \% \\
\text { a lesões por pressão e } 11,4 \% \text { a } \\
\text { quedas. A maioria dos eventos } \\
\text { ocorreu no período da manhã, nas } \\
\text { Unidades de Terapia Intensiva e } \\
\text { envolveu auxiliares/técnicos de En- } \\
\text { fermagem, seguidos pelo enfermeiro } \\
\text { e o médico. Em } 66,5 \% \text {, a causa raiz } \\
\text { predominante foi a falha do profis- } \\
\text { sional, principalmente, no processo } \\
\text { de comunicação nas transições do } \\
\text { cuidado. }\end{array}$ & IV \\
\hline
\end{tabular}




\begin{tabular}{|c|c|c|c|c|c|c|}
\hline$\frac{m}{\frac{m}{2}}$ & $\begin{array}{l}\text { Revista Gaúcha } \\
\text { de Enfermagem, } \\
\text { Brasil, } 2016\end{array}$ & $\begin{array}{c}\text { Alves DFS, } \\
\text { Guirardello EB }\end{array}$ & $\begin{array}{l}\text { Ambiente de traba- } \\
\text { lho da enfermagem, } \\
\text { segurança do } \\
\text { paciente e qualidade } \\
\text { do cuidado em } \\
\text { hospital pediátrico }\end{array}$ & $\begin{array}{c}\text { Estudo descritivo com } 136 \\
\text { profissionais de enfermagem } \\
\text { de um hospital pediátrico, } \\
\text { com aplicação da ficha de } \\
\text { caracterização pessoal e pro- } \\
\text { fissional, Nursing Work Index } \\
\text { - Revised, Safety Attitudes } \\
\text { Questionnaire - Short form } \\
2006 \text { e dos indicadores de } \\
\text { qualidade. }\end{array}$ & $\begin{array}{l}\text { Os profissionais percebem o } \\
\text { ambiente como favorável à prática } \\
\text { profissional, avaliaram como boa a } \\
\text { qualidade do cuidado e a redução } \\
\text { de eventos adversos e da per- } \\
\text { manência hospitalar. O domínio } \\
\text { satisfação no trabalho foi favorável } \\
\text { a segurança do paciente. }\end{array}$ & IV \\
\hline$\frac{\sqrt{\frac{\pi}{0}}}{\frac{0}{2}}$ & $\begin{array}{c}\text { Revista Mineira } \\
\text { de Enfermagem, } \\
\text { Brasil, } 2015\end{array}$ & $\begin{array}{l}\text { Siqueira CL, Silva } \\
\text { CC, Teles JKN, } \\
\text { Feldman LB }\end{array}$ & $\begin{array}{l}\text { Gerenciamento de } \\
\text { risco: percepção de } \\
\text { enfermeiros em dois } \\
\text { hospitais do Sul de } \\
\text { Minas Gerais, Brasil }\end{array}$ & $\begin{array}{c}\text { Estudo com abordagem quali- } \\
\text { tativa, de caráter exploratório, } \\
\text { descritivo, com } 29 \text { enfermeiros } \\
\text { assistenciais e gerenciais } \\
\text { de dois hospitais privados e } \\
\text { de grande porte localizado } \\
\text { no interior de Minas Gerais. } \\
\text { Os dados foram submeti- } \\
\text { dos à técnica de análise de } \\
\text { conteúdo. }\end{array}$ & $\begin{array}{l}\text { Os resultados mostraram que os } \\
\text { enfermeiros relacionam o gerencia- } \\
\text { mento de risco como ferramenta de } \\
\text { qualidade e segurança na assistên- } \\
\text { cia ao paciente. }\end{array}$ & IV \\
\hline 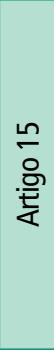 & $\begin{array}{c}\text { Escola Anna Nery } \\
\text { Revista de Enfer- } \\
\text { magem, Brasil, } \\
2014\end{array}$ & $\begin{array}{l}\text { Oliveira RM, Lei- } \\
\text { tão IMTA, Silva } \\
\text { LMS, Figueiredo } \\
\text { SV, Sampaio RL, } \\
\text { Gondim MM }\end{array}$ & $\begin{array}{l}\text { Estratégias para } \\
\text { promover segurança } \\
\text { do paciente: da iden- } \\
\text { tificação dos riscos } \\
\text { às práticas baseadas } \\
\text { em evidências }\end{array}$ & $\begin{array}{l}\text { Estudo descritivo, qualitativo, } \\
\text { desenvolvido em hospital } \\
\text { público de Fortaleza-CE. A } \\
\text { coleta de dados procedeu-se } \\
\text { mediante entrevista semies- } \\
\text { truturada com } 37 \text { enfermeiros, } \\
\text { analisada segundo o referen- } \\
\text { cial da análise de conteúdo. }\end{array}$ & $\begin{array}{l}\text { Os participantes identificaram riscos } \\
\text { físicos/químicos, clínicos, assis- } \\
\text { tenciais e institucionais, além de } \\
\text { barreiras e oportunidades que impli- } \\
\text { cam na (in)segurança do paciente. } \\
\text { Por outro lado, referiram práticas } \\
\text { embasadas em metas internacio- } \\
\text { nais divulgadas pela Organização } \\
\text { Mundial de Saúde. }\end{array}$ & IV \\
\hline$\frac{0}{\frac{8}{2}}$ & $\begin{array}{l}\text { Acta Paulista de } \\
\text { Enfermagem, } \\
\text { Brasil, } 2014\end{array}$ & $\begin{array}{l}\text { Teixeira TCA, } \\
\text { Cassiani SHB }\end{array}$ & $\begin{array}{l}\text { Análise de causa } \\
\text { raiz de acidentes } \\
\text { por quedas e erros } \\
\text { de medicação em } \\
\text { hospital }\end{array}$ & $\begin{array}{l}\text { Trata-se de estudo transversal } \\
\text { e exploratório realizado com } \\
62 \text { incidentes notificados } \\
\text { no período de estudo. } 0 \\
\text { instrumento de pesquisa foi } \\
\text { elaborado para coletar dados } \\
\text { dos formulários de notificação } \\
\text { e dos prontuários dos pacien- } \\
\text { tes. A validação de conteúdo } \\
\text { do instrumento foi realizada } \\
\text { por juízes. Foram constituídas } \\
\text { duas equipes para análise } \\
\text { da causa raiz dos incidentes } \\
\text { e categorização dos fatores } \\
\text { causais. }\end{array}$ & $\begin{array}{l}\text { No período de estudo foram } \\
\text { notificados } 62 \text { incidentes, sendo } \\
11 \text { quedas e } 51 \text { erros de medica- } \\
\text { ção. A maior parte das quedas foi } \\
\text { da própria altura, e os principais } \\
\text { tipos de erros de medicação foram } \\
\text { omissão e horário. Dos } 19 \text { incidentes } \\
\text { analisados, um total de } 118 \text { fatores } \\
\text { causais foram identificados, sendo } \\
\text { a maioria relacionada às falhas } \\
\text { sistêmicas, seguidas por falhas do } \\
\text { indivíduo e do paciente. }\end{array}$ & IV \\
\hline
\end{tabular}

No que tange ao ano de publicação, dos dezesseis artigos analisados, foi constatado que a maior quantidade foi publicada no ano de 2017, 2018 e 2019, ambos com quatro artigos (25\%), seguido de $2014 \mathrm{com}$ dois $(12,5)$ e, por fim, 2015 e 2016 com um artigo (6,25\%). O periódico de maior publicação foi a Revista de Enfermagem da UFPE On line (REUOL), com o total de quatro artigos (25\%). Quanto a profissão da primeira autora dos artigos, em seis $(37,5 \%)$ a primeira autoria era de enfermeira, em um $(6,25 \%)$ era de estudante de enfermagem e em nove $(56,25 \%)$ não foram encontradas informações específicas relacionadas à sua formação, pois apresentava-se somente a vinculação acadêmica.

Quanto a região do país em que as pesquisas foram realizadas, onze $(68,75 \%)$ foram desenvolvidas na região Sudeste, três (18,75\%) na região Nordeste, uma $(6,25 \%)$ na região Sul, nenhuma $(0 \%)$ na região Centro-Oeste e apenas uma $(6,25 \%)$ foi realizada a nível nacional. 


\section{DISCUSSÃO}

Aborda-se no artigo 6 que a elevada taxa de pacientes acometidos por eventos adversos evitáveis demonstra a seriedade do problema e a premência por ações que minimizem a ocorrência de danos considerados desnecessários ao paciente ${ }^{14}$.

Ademais, a qualidade e segurança do cuidado não depende apenas de profissionais qualificados, existindo outros fatores que implicam na execução dos processos assistenciais como a estrutura adequada e os recursos materiais suficientes ${ }^{15}$. Nessa perspectiva, os sistemas de notificação de incidentes, com foco na segurança do paciente, têm sido vastamente utilizados pelas instituições, visando a identificação e a análise consistente dos eventos adversos ${ }^{16}$.

De acordo com os artigos 3, 10 e 16, a ocorrência dos eventos deve ser notificada, visto que ela é fonte de informações úteis para obter registros, detectar causas e através dela e da investigação e análise de incidentes poderão ser implementadas ações de mudanças para melhoria do cuidado, a fim de possibilitar um ambiente de trabalho seguro, sem riscos e baseado nas melhores práticas, e assim é possível atingir a segurança do paciente e a excelência na qualidade do serviço ${ }^{17-18,5}$.

No artigo 11 é afirmado que os eventos ocorridos levam a mudanças positivas para segurança e que é importante haver discussão de estratégias de prevenção que garantam que estes eventos não voltem a ocorrer ${ }^{19}$.

Com a aplicação da metodologia da análise de causa raiz, no artigo 16, as causas foram classificadas em relação aos fatores causais, sendo a maioria dos fatores identificados como falhas sistêmicas, seguidas por falhas do indivíduo e do paciente, confirmando que incidentes decorrem principalmente de falhas sistêmicas e não individuais ${ }^{18}$.

Os artigos 6, 12 e 16 apontaram como causas para ocorrência dos eventos adversos a sobrecarga de trabalho, a falha na comunicação tanto entre os profissionais da equipe como com o paciente, o ambiente físico inadequado, a dificuldade de acesso a normas e procedimentos operacionais descritos, a ausência de padronização das condutas no processo de transição do cuidado, a ausência de registro em prontuário, o horário próximo à passagem de plantão, o horário de maior demanda de trabalho e a ilegibilidade da prescrição médica ${ }^{14,18,20}$.

A dimensão estrutural das organizações de saúde, também é abordada nos artigos 1 e 5 que enfatizam a necessidade de mudanças estruturais, apontando a importância do ambiente adequado, da disponibilidade de recursos físicos, materiais e humanos para que o cuidado seja seguro, eficaz e sem danos ${ }^{15,21-22}$.

Sabe-se que o quantitativo apropriado da equipe de profissionais contribui para a qualidade da assistência. Todavia, pesquisas indicam que ainda hoje a escala de pessoal não é suficiente para as demandas de cuidado. A questão do dimensionamento inadequado de pessoal é mostrada nos $\operatorname{artigos}^{7,8,9,12}$, 14 e 15 como fator impactante para ocorrência de incidentes, visto que a sobrecarga de trabalho e a superlotação são prejudiciais à execução das atividades dos enfermeiros e emergem condições inseguras. Dessa maneira evidencia-se a necessidade de priorizar investimentos para adequações físicas e estruturais nas instituições ${ }^{16,20,23-26}$.

No artigo 4, evidencia-se que o estresse e as condições de trabalho são itens que comprometem a qualidade da assistência ${ }^{27}$. Outro estudo corrobora para esse achado e menciona o estresse como um elemento que pode prejudicar a organização do trabaIho e acarretar em evento adverso ${ }^{20}$.

A jornada de trabalho por vezes dupla vivenciada pela maioria destes profissionais, embora tenha os benefícios relacionados ao aumento da renda, de certo modo, acaba reduzindo o tempo dedicado ao autocuidado e ao lazer, potencializando o cansaço e o estresse e assim, facilitando a ocorrência dos erros ${ }^{23,26}$.

Todavia, constatou-se nos artigos 11 e 14, que muitas vezes há subnotificação devido a alta rotatividade, a falta de tempo, ao medo de punição e a existência de medidas punitivas aplicadas pelas instituições diante da ocorrência de even- to adverso ${ }^{16,19}$. Tal realidade interfere na análise dos riscos e eventos ocorridos e na elaboração de melhorias e compromete a efetividade dos cuidados de enfermagem e o seu gerenciamento de modo seguro ${ }^{16}$.

É apontado que o sucesso de um sistema de notificação necessita da eliminação do paradigma de ocultação do erro relacionado à preocupação com o fato de o registro poder ser utilizado contra o próprio profissional, contrariando a nova cultura a ser adotada nas instituições de saúde, a fim de tornar a notificação uma ação voluntária de fato, pois sabe-se que um ambiente organizado e livre da cultura punitiva assegura o sucesso na assistência segura ao paciente ${ }^{16,18}$.

Nessa perspectiva, os artigos 10 e 16 reforçam que para vencer lacunas no conhecimento e aumentar o comprometimento na identificação e redução de EA é necessário adotar condutas que enrijeça essa cultura de segura e postura não punitiva, pois frequentemente o medo da punição implica na subnotificação dos incidentes ocorridos ${ }^{5,18,28}$.

Enfim, compreender a relação entre os riscos, as características dos cuidados à saúde e os recursos humanos, materiais e físicos e a importância da notificação dos eventos adversos como ferramenta para monitorização dos incidentes, pode fornecer elementos importantes para promoção da segurança e da qualidade na assistência à saúde, dado o entendimento que existem lacunas no processo de cuidar.

\section{CONCLUSÃO}

Conclui-se que a notificação dos eventos adversos é um importante instrumento de gestão e meio de aprendizagem para a melhoria contínua dos processos assistenciais, e fundamental para análise dos incidentes, identificação das possíveis causas e implantação de estratégias que previnam a reincidência dos erros evitáveis.

Revelam-se alguns elementos que podem interferir na qualidade da assistência e contribuir para ocorrência de falhas no processo de trabalho, comprometendo a segurança do paciente, como os recursos físicos e humanos inadequados. 
Acredita-se que o estudo contribuiu para apresentar lacunas que prejudicam a oferta de um cuidado seguro, além de enfatizar a importância da notificação de EA.

Quanto as limitações, estas devem-se à própria metodologia de pesquisa, visto que a revisão integrativa da literatura possibilita pouca generalização dos resultados, por se tratar de estudos com métodos de análise dos objetos e locais de desenvolvimento das pesquisas diferentes. No entanto, são estudos generalizáveis quanto as proposições teóricas.
Ademais, a heterogeneidade metodológica impediu a análise estatística com metanálise na avaliação dos fatores relacionados as contribuições da notificação de eventos adversos para a segurança do paciente e aos entraves no processo de trabalho.

\section{Referências}

1. Tase TH, Quadrado ERS, Tronchin DMR. Evaluation of the risk of misidentification of women in a public maternity hospital. Rev Bras Enferm [Internet]. 2018 [cited 2019 Nov 11];71(1):120-5. Available from: http://dx.doi.org/10.1590/0034-71672017-0134

2. Institute of Medicine. To err is human: building a safer health system. Washington: National Academic Press; 1999.

3. Lemos GC, Azevedo C, Bernardes MFVG, Ribeiro HCTC, Menezes AC, Mata LRF. The patient safety culture in the scope of nursing: theoretical reflextion. Rev Enferm Cent-Oeste Min [Internet]. 2018 [cited 2019 Nov 10];8:e2600. Available from: http://dx.doi.org/10.19175/recom.v8i0.2600

4. Faiella G, Parand A, Franklin BD, Chana P, Cesarelli M, Stanton NA et al. Expanding healthcare failure mode and effect analysis: a composite proactive risk analysis approach. Reliabil Eng Syst Safety [Internet]. 2018 [cited 2019 Nov 11];169:117-26. Available from: https://doi.org/10.1016/..ress.2017.08.003

5. SimanAG, CunhaSGS, Brito MJM. The practice of reporting adverse events in a teaching hospital. Rev Esc Enferm USP [Internet]. 2017 [cited 2019 Set 21];51:e03243. Available from: https://doi.org/10.1590/S1980-220X2016045503243

6. Costa DB, Ramos D, Gabriel CS, Bernardes A. Patient safety culture: evaluation by nursing professionals. Texto \& Contexto Enferm [Internet]. 2018 [cited 2019 Nov 11];27(3):e2670016. Available from: http://dx.doi.org/10.1590/0104070720180002670016

7. Rocha RPF, Pinho DLM. Patient safety in hemodialysis. Rev Enferm UFPE On line [Internet]. 2018 [cited 2019 Nov 11];12(12):3360-7. Available from: https://doi. org/10.5205/1981-8963-v12i12a235857p3360-3367-2018

8. Golle L, Ciotti D, Herr GEG, Aozone F, Schmidt CR, Kolankiewicz ACB. Culture of patient safety in hospital private. Rev Pesqui (Univ Fed Estado Rio J Online) [Internet]. 2018 [cited 2019 Nov 11];10(1):85-89. Available from: http://dx.doi. org/10.9789/2175-5361.2018.v10i1.85-89

9. Marinho MM, Radünz V, Rosa LM, Tourinho FSV, Ilha P, Misiak M. Education interventions with nursing professionals and its relationship with culture of securityh. REME Rev Min Enferm [Internet]. 2018 [cited 2019 Nov 11];22:e-1148. Available from: https://doi.org/10.5935/1415-2762.20180079

10. Furini ACA, Nunes AA, Dallora MELV. Notifications of adverse events: characterizations of the events that ocurred in a hospital complex. Rev Gaúch Enferm [Internet]. 2019 [cited 2019 Nov 11];40(esp):e20180317. Available from: https:/l doi.org/10.1590/1983- 1447.2019.20180317.

11. Aguiar RS, Santana DC, Santana PC. A percepção do enfermeiro da estratégia saúde da família sobre a saúde do homem. Rev Enferm Cent-Oeste Min [Internet]. 2015 [cited 2019 Jun 05];5(3):1844-54. Available from: https://doi.org/10.19175/ recom.v5i3.872

12. Donato $H$, Donato $M$. Etapas na condução de uma revisão sistemática. Acta Med Port [Internet]. 2019 [cited 2019 Set 22];32(3):227-235. Available from: https://doi.org/10.20344/amp.11923

13. Moher D, Liberati A, Tetzlaff J, Altman DG, The PRISMA Group. Preferred reporting items for systematic reviews and meta-analyses: the PRISMA statement. PLoS Med [Internet] 2009 [cited 2019 Set 24];6(7):e1000097. Available from: https://doi. org/10.1371/journal.pmed.1000097

14. Figueiredo ML, Oliveira e Silva CS, Brito MFSF, D'Innocenzo M. Analysis of incidents notified in a general hospital. Rev Bras Enferm [Internet]. 2018 [cited 2019 Set 21];71(1):111-9. Available from: https://doi.org/10.1590/0034-7167-20160574

15. Pereira MCC, Castro SFF, Brito ES, Carvalho NV, Lopes DV, Pinheiro JDS et al. Nurse's knowledge and practices in the intensive care unit. Rev Enferm UFPE On line [Internet]. 2019 [cited 2019 Set 19];13(1):70-8. Available from: https://doi.org/10.5205/1981-8963-v13i01a234842p70-78-2019

16. Siqueira CL, Silva CC, Teles JKN, Feldman LB. Management: perception of nurses of two hospitals in the South of the state of Minas Gerais, Brazil. REME Rev Min Enferm [Internet]. 2015 [cited 2019 Set 17];19(4):927-933. Available from: https:// doi.org/10.5935/1415-2762.20150071

17. Moraes AIS, Santos VL, Paes LBO, Parro MC. Qualidade e segurança na área da saúde materno infantil: avaliação de eventos adversos. CuidArte Enferm [Internet]. 2019 [cited 2019 Set 18];13(1):32-37. Available from: http://www.webfipa.net/facfipa/ner/sumarios/cuidarte/2019v1/32.pdf

18. Teixeira TCA, Cassiani SHB. Root cause analysis of falling acidentes and medication erros in hospital. Acta Paul Enferm (Online) [Internet]. 2014 [cited 2019 Set 19];27(2):100-7. Available from: https://doi.org/10.1590/19820194201400019 19. Ferezin TPM, Ramos D, Caldana G, Gabriel CS, Bernardes A. Analysis of adverse event reporting at accredited hospitals. Cogitare Enferm [Internet]. 2017 [cited 2019 Set 19]; (22)2:e49644. Available from: https://doi.org/10.5380/ce v22i2.49644

20. Pena MM, Melleiro MM. The root cause analysis method for the investigation of adverse events. Rev Enferm UFPE On line [Internet]. 2017 [cited 2019 Set 17];11(supl.12):5297-304. Available from: https://doi.org/10.5205/1981-8963-v11 i12a25092p5297-5304-2017

21. Gomes ATL, Ferreira Júnior MA, Salvador PTCO, Bezerril MS, Chiavone FBT, Santos VEP. Safety of the patient in an emergency situation: perceptions of the nursing team. Rev Bras Enferm [Internet]. 2019 [cited 2019 Set 22];72(3):753-9. Available from: https://doi.org/10.1590/0034-7167-2018-0544

22. Gutierres LS, Santos JLG, Peiter CC, Menegon FHA, Sebold LF, Erdmann AL, Good practices for patient safety in the operating room: nurses' recommendations. Rev Bras Enferm [Internet]. 2018 [cited 2019 Set 22];71(suppl.6):2775-82. Available from: https://doi.org/10.1590/0034-7167-2018-0449

23. Mazzoni VG, Bittencourt LP, Ribeiro ML, Gouvêa MV. Challenges of the organizational dimension of care in the daily work of nursing workrs. Rev Enferm UFPE On line [Internet]. 2018 [cited 2019 Set 17];12(1):11-8. Available from: https://doi. org/10.5205/1981-8963-v12i01a230392p11-18-2018

24. Ortega DB, D'Innocenzo M, Silva LMG, Bohomol E. Analysis of adverse events in patients admitted to na intensive care unit. Acta Paul Enferm (Online) [Internet]. 2017 [cited 2019 Set 21];30(2):168-73. Available from: https://doi. org/10.1590/19820194201700026

25. Silva AT, Camelo SHH, Terra FS, Dázio EMR, Sanches RS, Resck ZMR. Patient safety and the nurse's performance in hospital. Rev. enferm. UFPE on line. [Internet] 2018 [cited 2019 Set 18];12(6):1532-8. Available from: https://doi.org/10.5205/ 1981-8963-v12i6a234593p1532-1538-2018

26. Oliveira RM, Leitão IMTA, Silva LMS, Figueiredo SV, Sampaio RL, Gondim MM. Strategies for promoting patient afety: from the identification of the risks to the evidence-based practices. Esc Anna Nery Rev Enferm [Internet]. 2014 [cited 2019 Set 20];18(1):122-129. Available from: https://doi.org/10.5935/1414-8145.20140018 27. Picolotto A, Barella D, Moraes FR, Gasperi P. The patienty safety culture of a nursing team from a central ambulatory. Rev Pesqui (Univ Fed Estado Rio J Online) [Internet]. 2019 [cited 2019 Set 18];11(esp):333-338. Available from: https://doi. org/10.9789/21755361.2019.v11i2.333-338

28. Alves DFS, Guirardello EB. Nursing work environment, patient safety and quality of care in pediatric hospital. Rev Gaúch Enferm [Internet]. 2016 [cited 2019 Set 20];37(2):e58817. Available from: https://doi.org/10.1590/19831447.2016.02.58817 